\title{
BMJ Open Development of patient decision support tools for motor neuron disease using stakeholder consultation: a study protocol
}

\author{
Anne Hogden, ${ }^{1}$ David Greenfield, ${ }^{1}$ Jashelle Caga, ${ }^{2}$ Xiongcai Cai ${ }^{3}$
}

To cite: Hogden A, Greenfield D, Caga J, et al. Development of patient decision support tools for motor neuron disease using stakeholder consultation: a study protocol. BMJ Open 2016;6:e010532.

doi:10.1136/bmjopen-2015010532

- Prepublication history for this paper is available online. To view these files please visit the journal online (http://dx.doi.org/10.1136/ bmjopen-2015-010532).

Received 12 November 2015 Revised 29 February 2016 Accepted 21 March 2016

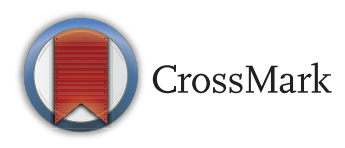

${ }^{1}$ Centre for Healthcare Resilience and Implementation Science, Australian Institute of Health Innovation, Macquarie University, Sydney, New South Wales, Australia ${ }^{2}$ Sydney Medical School, University of Sydney, Sydney, New South Wales, Australia ${ }^{3}$ School of Computer Science and Engineering, University of New South Wales, Sydney, New South Wales, Australia

Correspondence to Dr Anne Hogden; anne.hogden@mq.edu.au

\section{ABSTRACT}

Introduction: Motor neuron disease (MND) is a terminal, progressive, multisystem disorder. Well-timed decisions are key to effective symptom management. To date, there are few published decision support tools, also known as decision aids, to guide patients in making ongoing choices for symptom management and quality of life. This protocol is to develop and validate decision support tools for patients and families to use in conjunction with health professionals in MND multidisciplinary care. The tools will inform patients and families of the benefits and risks of each option, as well as the consequences of accepting or declining treatment.

Methods and analysis: The study is being conducted from June 2015 to May 2016, using a modified Delphi process. A 2-stage, 7-step process will be used to develop the tools, based on existing literature and stakeholder feedback. The first stage will be to develop the decision support tools, while the second stage will be to validate both the tools and the process used to develop them. Participants will form expert panels, to provide feedback on which the development and validation of the tools will be based. Participants will be drawn from patients with MND, family carers and health professionals, support association workers, peak body representatives, and MND and patient decision-making researchers.

Ethics and dissemination: Ethical approval for the study has been granted by Macquarie University Human Research Ethics Committee (HREC), approval number 5201500658. Knowledge translation will be conducted via publications, seminar and conference presentations to patients and families, health professionals and researchers.

\section{INTRODUCTION}

Motor neuron disease (MND), also known as amyotrophic lateral sclerosis, is a terminal, progressive, multisystem disorder. Incidence worldwide is estimated at 2-3 per 100000 of total population. ${ }^{1}$ Approximately 1500 people are currently living with MND in

\section{Strengths and limitations of this study}

- A user-centric approach to decision support tool development will result in tools that address the needs of patients with motor neuron disease (MND), carers and health professionals.

- As the modified Delphi process is iterative and consultative, consensus between panel members may take time to achieve.

- A comprehensive review of published and grey literature in the MND and patient decisionmaking fields will be undertaken. However, this will not be conducted as a systematic literature review.

Australia. There is, as yet, no cure, and treatment options offer little extension to life expectancy. The cause of the disease remains unknown. MND is a heterogenic condition, with a range of clinical presentations, or phenotypes. ${ }^{2}{ }^{3}$ Survival time varies with phenotype, averaging around 3 years. ${ }^{4}$ Death most frequently results from respiratory failure. ${ }^{5}$ Patients experience physical deterioration that frequently affects their mobility, swallowing and speech. Deterioration of patients' physical status may be accompanied by changes to their cognitive skills and behaviour. ${ }^{6}$ Around $50 \%$ of patients are thought to be affected by mild cognitive or behavioural change. ${ }^{7}$ Cognitive change may present as frontotemporal dementia or, more frequently, as mild cognitive change affecting capacity to control and regulate cognitive process such as memory and information processing skills. ${ }^{2-8}$ Behavioural change is characterised by apathy and impulsivity. ${ }^{8}$ The complexity of MND creates a challenging environment for patient care.

Treatment approaches for this complex condition are palliative in nature. They include disease-slowing medication and artificial support for respiration, feeding and 
hydration. Care received through specialised multidisciplinary clinics has also been demonstrated to promote survival. ${ }^{9}$ Patients and families are typically able to access a range of medical, nursing, allied health clinicians and support association staff within the multidisciplinary clinic during a single visit. Patients with MND and families are asked to make numerous decisions for symptom management and quality of life as the disease progresses. These decisions often involve consultation with a range of health professionals before patients decide how they wish to proceed. ${ }^{10}$ Moreover, due to the high burden of physical care and emotional support required, family members are frequently involved in patients' decision-making process. ${ }^{11}$ Patients' decision-making is complicated by ongoing deterioration, psychological distress, changing psychosocial circumstances, ${ }^{12}$ and defined time frames for medical and surgical intervention. Decisions are often delayed when patients and families experience difficulty engaging with health professionals to discuss symptom management. ${ }^{13}$

The progressive nature of MND, and the range of physical and intellectual functions it compromises, creates an urgency for patients and families to understand, and respond to, complex medical and health service information. Patients and family members favour healthcare information from sources they trust, such as MND support associations, health professionals and research-based websites. ${ }^{11}$ Moreover, health professionals support the use of evidence-based information sources to assist patients to make realistic and informed decisions. ${ }^{14}$ Not surprisingly, patients with MND and families report feeling overwhelmed by the amount and confronting nature of information required for symptom management decisions. ${ }^{11-15}$ However, poorly timed decisions compromise patient health, safety and quality of life, increase carer burden, and can result in costly and distressing emergency hospital admissions. ${ }^{16}$ In these circumstances, the stress for patients, carers and health professionals is significant, and potential for problems and less than optimal care increases. ${ }^{17}$

Decision support tools, also known as decision aids, are used in a range of healthcare conditions ${ }^{18}$ to guide patients through treatment decisions. Typically, such tools have four components. They summarise evidence-informed and best practice options; present patients and families with the risks and benefits of the choices available to them; ${ }^{19}$ check patients' understanding of their options; and clarify personal values and preferences that influence choices, such as use of treatments artificially extending life. Tools assist patients to discuss their options with family and health professionals, understand the consequences of accepting or declining treatment, and make informed choices for care. There is also potential to facilitate safer and more cost-effective care. ${ }^{16}$ By further improving patient participation in care discussions, use of the tools may optimise the timing and effectiveness of care decisions. ${ }^{20}$ When used during a care consultation, tools facilitate communication between patients, family members and health professionals, as well as allowing clinicians to tailor tools and decisions to patients' individual circumstances. $^{21}$

Currently, in the MND field, there are few decision support tools providing structured guidance to patients and family carers for symptom management and quality of life considerations. Owing to the unique MND disease characteristics and trajectory, none of the existing tools from deteriorating conditions such as cancer ${ }^{22}$ or chronic disease $\mathrm{e}^{23}$ are appropriate for symptom management. As MND is a terminal condition lacking a cure, and with limited disease-delaying options, decisionmaking frequently becomes a choice between a single treatment option, or doing nothing. MND decision support tools need to inform patients on key decisions that relate specifically to MND management, such as the use of disease-slowing medication, the timing windows of gastrostomy insertion in rapidly progressive disease. While many of the equipment and procedures used to manage MND are common to other conditions, the timing, risks and benefits are very specific to the individual's disease course. Moreover, there is little evidence available to support most of the options. The value of these tools is in preparing patients to consider their values and preferences as they encounter disease milestones.

By drawing on stakeholder consultation, user-centred design principles, ${ }^{24}$ and guided by the International Patient Decision Aids Standards (IPDAS), ${ }^{25}$ this project will develop evidence-informed MND-specific tools to support patients' key symptom management and quality of life decisions. ${ }^{26} 27$ Tools may include use of diseaseslowing medication, respiration support, artificial nutrition and hydration, saliva management, and end-of-life care. $^{28} 29$ The study aims are to produce decision support tools to meet the needs of patients with MND attending Australian multidisciplinary clinics. The tools will be designed as encounter tools; that is, tools for use in a multidisciplinary clinic appointment between patient, health professional and family carers. The purpose of the tools is to support patients to clarify their information needs, values and preferences before their decision is made. We will examine the following research questions (RQ):

RQ1. Which decision-making tools are needed to support patients with MND?

RQ2. What is the optimal content and format of these tools?

\section{METHODS AND ANALYSIS}

This research protocol details the process of development and validation of decision support tools for specialised MND multidisciplinary care. The study will be conducted over 12 months, from June 2015 to May 2016. Information to develop and validate decision support tools will be gathered from published and grey 
literature, and through a modified Delphi process. ${ }^{30}$ Expert panels will be formed from participants with experience in MND and/or patient decision-making. The deliberation and development process will be conducted electronically to include stakeholders from a range of locations, and to create an open forum for discussion between participants. The decision tools will be underpinned by research literature, and developed according to feedback received from the expert panels.

\section{Participants}

Participants will be recruited from seven stakeholder groups key to MND and patient decision-making, ${ }^{31}$ to form two expert panels, one for patients and carers, and one for health professionals and researchers. Use of separate panels will ensure patient and carer views on the decision support tools are independent clinician and researcher views. Views of the two panels will be integrated to contribute to tool development. The role of panel members will be to provide insight on key treatment and quality of life decisions. To optimise stakeholder representation, panel members will be sought purposively, to contribute according to their experience or expertise in MND or patient decision-making.

Potential participants will include patients with midstage or late-stage disease who have experienced decision-making for their care; and family members of current or deceased patients who were involved in the patient's decision-making. Patients experiencing mild cognitive and behavioural change, or early-stage frontotemporal dementia, who are able to give informed consent (as advised by clinicians and advisors from the recruitment settings) will be invited to participate. Inclusion of patients with cognitive and behavioural change will allow their views to shape the tools for the requirements of this clinically diverse population. However, patients with advanced frontotemporal dementia who are unable to give informed consent will be excluded from the study. Their carers will be invited to give perspective on the needs of this subgroup.

Participants for the health professional and researcher panel experienced health professionals working in MND multidisciplinary care; MND regional advisors with extensive experience in supporting patients and families; representatives from the MND peak body (MND Australia); MND clinical care researchers; and researchers with expertise in patient decision-making and decision support tool development. Researchers will include a human factors engineer to ensure that the tools are developed to meet users (ie, patients, carers and health professionals) physical and psychological needs. ${ }^{24}$

\section{Recruitment}

Recruitment will be conducted through three healthcare and research networks. First, a metropolitan specialised MND multidisciplinary clinic will be accessed by the study team. Patients with MND, carers and health professionals will be approached in person, and invited to participate. Second, patients, carers and MND support workers and a peak body representative will be recruited through MND New South Wales (MND NSW) and MND Australia. A recruitment letter will be forwarded to the association inviting staff to take part in the project. MND NSW staff will be asked to pass on study information to patients and carers they consider suitable to participate in the project. Third, researchers from the fields of MND clinical care and patient decision-making will be contacted by email through the study team's international research networks. All participants will be asked to give written consent prior to participation on the expert panel, and be available to complete assessments and respond to discussion topics during the study period.

\section{Expert panel consultation process \\ Overview}

The project will be conducted using a two-stage modified Delphi process of development and validation (table 1). As encounter tools, they will be designed for use by patients, carers and health professionals within clinical appointments at specialised MND multidisciplinary clinics. The process will involve iterative consultation, expected to involve a minimum of four cycles, to optimise participant feedback. Participants will first be asked to nominate decision tool topics for clinical care they consider most useful. These will be developed into drafts by the study team. Panel members will then be provided with tool drafts and asked to provide feedback. The feedback will be used to refine the tools, which will then be returned to panel members for further comment and refinement. Once the panel agrees that the draft is complete, the panel will validate the tools, and a prototype will be finalised by the study team. The development and validation stages of the project are described in detail below.

\section{Stage 1: tool development}

The tool development stage will consist of four steps. Step A will be a literature review to compile evidencebased and best practice information on MND symptom management, and decision tool development processes. ${ }^{32}$ Healthcare databases, including MEDLINE, the Cochrane Database, PsychINFO and grey literature, will be searched, using terms that capture key constructs of the study. Terms will include decision support tools, decision aids, choice behaviour, shared decision making, health communication, motor neuron(e) disease, amyotrophic lateral sclerosis and neurodegenerative disease. Patient information literature, such as that provided by MND associations and health policy documents, will also be sourced.

The tools will be checked against the IPDAS to ensure tools comply with international best practice standards as they are developed. ${ }^{25}$ The IPDAS criteria provide a framework to evaluate both the content of decision tools and the processes that are used to develop them. Moreover, the criteria emphasise stakeholder 
Table 1 Tool development and validation process summary

\begin{tabular}{|c|c|c|c|c|}
\hline Step & Objective & Method & $\begin{array}{l}\text { Timeline } \\
\text { (months) }\end{array}$ & Process \\
\hline \multicolumn{5}{|c|}{ Stage 1: tool development } \\
\hline A & $\begin{array}{l}\text { 1. Identify evidence-informed } \\
\text { and best practice } \\
\text { information for MND } \\
\text { symptom management } \\
\text { and quality of life issues } \\
\text { 2. Identify decision tool } \\
\text { development processes }\end{array}$ & $\begin{array}{l}\text { Literature review of: } \\
\text { MND symptom management } \\
\text { Quality of life issues } \\
\text { Decision tool development } \\
\text { processes } \\
\text { User-centred design }\end{array}$ & $1-4$ & $\begin{array}{l}\text { Determine evidence base and user } \\
\text { perspectives from literature } \\
\text { Gain ethics approval }\end{array}$ \\
\hline B & Form expert panel & $\begin{array}{l}\text { Recruitment and selection of } \\
\text { expert panel members from } \\
\text { stakeholder groups } \\
\text { Identify user priority list of } \\
\text { tools to be developed }\end{array}$ & $4-5$ & $\begin{array}{l}\text { Education of panel members on } \\
\text { participation, role requirements and } \\
\text { IPDAS criteria }\end{array}$ \\
\hline $\mathrm{C}$ & Development of tool drafts & $\begin{array}{l}\text { Expert panel consultation } \\
\text { Delphi process }\end{array}$ & $5-9$ & $\begin{array}{l}\text { Nomination of five highest priority } \\
\text { tools } \\
\text { Develop tool drafts } \\
\text { Iterative consultation with panel } \\
\text { members as development } \\
\text { progresses } \\
\text { Feedback loop with panel } \\
\text { members }\end{array}$ \\
\hline $\mathrm{D}$ & Final review of draft tools & $\begin{array}{l}\text { Expert panel review } \\
\text { Evaluation against IPDAS } \\
\text { criteria }\end{array}$ & 10 & $\begin{array}{l}\text { Consultation with panel members } \\
\text { IPDAS checklist }\end{array}$ \\
\hline \multicolumn{5}{|c|}{ Stage 2: validation process } \\
\hline$E$ & Validation of tool prototypes & $\begin{array}{l}\text { Expert panel agreement } \\
\text { Study team consensus }\end{array}$ & $10-11$ & $\begin{array}{l}\text { Signoff by panel members } \\
\text { Study team discussion }\end{array}$ \\
\hline $\mathrm{F}$ & $\begin{array}{l}\text { Refinement and validation of } \\
\text { tool development process }\end{array}$ & $\begin{array}{l}\text { Feedback from panel } \\
\text { members on development } \\
\text { process } \\
\text { Documentation of } \\
\text { development process } \\
\text { Validation of process by expert } \\
\text { panel and study team }\end{array}$ & 11 & $\begin{array}{l}\text { Formal mapping and } \\
\text { documentation of the development } \\
\text { process actually used in the study. } \\
\text { A draft of this document will be } \\
\text { circulated to the expert panel. } \\
\text { The expert panel will be asked to } \\
\text { give feedback on ways the process } \\
\text { could have been improved. } \\
\text { The process will then be refined } \\
\text { according to participant feedback. } \\
\text { The process will be documented } \\
\text { for knowledge translation. }\end{array}$ \\
\hline G & Knowledge translation & $\begin{array}{l}\text { Reporting to HREC } \\
\text { Reporting to funding body } \\
\text { Feedback to participants } \\
\text { Publication of study findings }\end{array}$ & 12 & $\begin{array}{l}\text { Written reports } \\
\text { Newsletter to participants via email } \\
\text { Peer-reviewed journal publication } \\
\text { - Seminar presentations }\end{array}$ \\
\hline
\end{tabular}

HREC, Human Research Ethics Committee; IPDAS, International Patient Decision Aids Standards; MND, motor neurone disease.

involvement in development and testing. Formats suitable for patient use within the MND multidisciplinary clinic setting will be determined, that is, paper $^{34}$ and electronic $^{35}$ formats. Paper tools will follow the Ottawa Hospital Research Institute (OHRI) guidelines, ${ }^{36}$ while electronic tools will be guided by similar exemplar formats that are certified by IPDAS. The feasibility of phone app $^{37}$ formats will also be scoped by the study team. A period of up to 4 months has been allocated to this step.
Step B will be to form the expert panel, using the participant recruitment process described above. Once the panel has been assembled, members will be provided with further education on the aims of the project, their roles, and timeframes of the consultation and feedback process. Participants will be informed about the use of IPDAS criteria to ensure the evidence base, quality and relevance of decision support tools. Forming and educating the expert panel is expected to take up to 2 months. 
Step C is the development of the highest priority tools into draft form. Discussions with expert panel members will begin immediately after recruitment. This is to ensure that patients, carers and health professionals, as users of decision support tools, are included in a feedback loop from the beginning of the development process. Once participants have been educated about project requirements, the panel will be asked to generate a list of tools that they consider high priority to MND clinical care, aligned with IPDAS criteria and evidence-based literature.

A modified Delphi process will be applied, asking the panel to nominate the five most useful tools for clinical care, from user perspectives. ${ }^{33}$ These five tools will be developed into paper drafts, by an iterative process of review and refinement. It is anticipated that four cycles per tool will be necessary. A period of 1 week is allowed for each cycle. Expert panel members will be sent tool drafts by email, and asked to provide feedback in line with their experience and expertise. Electronic communication will enable regular, documented group comment on each draft. Participants will be asked to reply to all the members of the panel and study team to create an open forum for feedback. To facilitate and structure comments, the study team will use a discussion guide, based on the literature and the study team's clinical expertise. Participants will be asked to comment on issues of: design; information content; readability; format; and optimal timing of use between patients, carers and health professionals. Five months has been allocated for the production of draft tools.

Step D will be conducted once the five draft tools have been completed. The expert panel and study team will be asked to give a final review of the drafts, and check their content and development against the IPDAS criteria. Group discussion will be used to resolve disputes, solve problems and reach consensus on final drafts. Once agreement has been reached on the content and format of the tools, the development stage of the drafts will be complete. From this point, the tools will be termed prototypes. A period of up to 1 month has been set aside to complete this step.

\section{Stage 2: tool validation}

In step E, the expert panel will be asked to validate tool prototypes by certifying that the prototypes are appropriate for testing in the MND multidisciplinary care clinical environment. Panel agreement on the prototypes will be documented. The study team will also be asked to reach consensus on the prototypes, using the group discussion process described in step D. Once agreement is reached, the prototypes will be considered validated. Two weeks has been set aside for this step.

Refinement and validation of the process used to develop the decision tools occurs in step F, over the period of a month. The development process used in the study will be formally documented, and a draft will be circulated to the expert panel for feedback on ways the process could have been improved. The process will be refined according to participant feedback, and documented for dissemination.

Step $G$ is the final stage of development and validation, by knowledge translation. Taking place in the final month of the project, knowledge dissemination and translation will be conducted reporting to the funding body and the Macquarie University Human Research Ethics Committee (HREC). Journal articles will be prepared for peer review, and seminar presentations will be organised for academic and industry audiences. The peer review process will further contribute to the validation of the tool prototypes and development process.

\section{ETHICS AND DISSEMINATION}

Ethical approval for the study has been granted by Macquarie University HREC, approval number 5201500658. Recruitment flyers and information sheets summarising the study and the role of participants, as well as consent forms, were also approved. Management of participant information will be conducted in accordance with Macquarie University guidelines; that is, all personal information will be de-identified and kept in password-protected electronic files. Information will be destroyed after a minimum of 7 years.

Research dissemination and knowledge translation will be conducted in three ways. Participants will be provided with a summary of the process and outcomes of the research. Results will be published in peer-reviewed publications, targeting healthcare service and decisionmaking journals. Conference and seminar presentations will be given to the Australian and international MND research community, in accordance with funding body agreement. Subject to funding availability, this study will be extended for further research. Future projects aim to implement the decision support tools in a range of MND multidisciplinary clinics, evaluating the clinical feasibility of the tools, and developing guidelines for use in MND clinical care. Broader stakeholder input and peer review will provide robust validation.

Decision support tools developed for MND care have wider clinical application. At the conclusion of this project, the study team will explore avenues to develop decision support tools for a range of degenerative neurological conditions, including multiple sclerosis, Parkinson's disease and brain cancer. While specific symptom management options will not be directly applicable to other disease groups, the user-centric process for developing the tools and formatting for multidisciplinary clinical care will be adaptable for a range of neurological conditions that currently lack decision support. The project has potential to be expanded to countries and language groups beyond the Australian context.

The innovation of this research is the production of purpose-designed tools to improve MND patient care. 
Table 2 Anticipated benefits for MND practice and policy

\begin{tabular}{|c|c|}
\hline $\begin{array}{l}\text { Stakeholder } \\
\text { group }\end{array}$ & Benefit \\
\hline Patients & $\begin{array}{l}\text { Improved engagement of patients and } \\
\text { families in care } \\
\text { Improved patient health literacy } \\
\text { Timely and well-informed treatment } \\
\text { decisions, leading to proactive symptom } \\
\text { management }\end{array}$ \\
\hline $\begin{array}{l}\text { Health } \\
\text { professionals }\end{array}$ & $\begin{array}{l}\text { Implementation of proactive approach to } \\
\text { symptom management } \\
\text { Enhancement of multidisciplinary team } \\
\text { care } \\
\text { Knowledge transfer of clinically useful } \\
\text { information } \\
\text { - Promotion of evidence-based practice }\end{array}$ \\
\hline $\begin{array}{l}\text { Health } \\
\text { services }\end{array}$ & $\begin{array}{l}\text { Improved organisational efficiency } \\
\text { Cost savings from reduced emergency } \\
\text { admissions } \\
\text { Improved use of resources } \\
\text { Knowledge transfer from research } \\
\text { directly to organisations }\end{array}$ \\
\hline $\begin{array}{l}\text { Healthcare } \\
\text { policy }\end{array}$ & $\begin{array}{l}\text { Links policy to clinical work } \\
\text { Incorporation of evidence-based } \\
\text { research into policy } \\
\text { Tools incorporate policy unique to each } \\
\text { country }\end{array}$ \\
\hline $\begin{array}{l}\text { Research } \\
\text { community }\end{array}$ & $\begin{array}{l}\text { Consolidation of international MND } \\
\text { research community } \\
\text { Support for international MND } \\
\text { healthcare community } \\
\text { Decision tool dissemination to other } \\
\text { deteriorating neurological conditions }\end{array}$ \\
\hline
\end{tabular}

The study has a multidimensional goal to advance patient care, health professional teamwork, service delivery and policy development. The project seeks to provide five significant healthcare and research benefits, within Australia and internationally (table 2).

Twitter Follow Anne Hogden at @annehogden

Contributors $\mathrm{AH}$ and DG designed the study, and prepared the manuscript. JC and XC contributed to the conceptual development of the study. All authors contributed to revisions of the manuscript.

Funding This work was supported by Motor Neurone Disease Research Institute of Australia MND Victoria Research Grant, grant number GIA 1525.

Competing interests None declared.

Ethics approval Ethical approval for the study has been granted by Macquarie University Human Research Ethics Committee (HREC), approval number 5201500658.

Provenance and peer review Not commissioned; externally peer reviewed.

Data sharing statement Data available on request from the corresponding author.

Open Access This is an Open Access article distributed in accordance with the Creative Commons Attribution Non Commercial (CC BY-NC 4.0) license, which permits others to distribute, remix, adapt, build upon this work noncommercially, and license their derivative works on different terms, provided the original work is properly cited and the use is non-commercial. See: http:// creativecommons.org/licenses/by-nc/4.0/

\section{REFERENCES}

1. Kiernan MC, Vucic S, Cheah BC, et al. Amyotrophic lateral sclerosis Lancet 2011;377:942-55.

2. Strong MJ, Grace GM, Freedman M, et al. Consensus criteria for the diagnosis of frontotemporal cognitive and behavioural syndromes in amyotrophic lateral sclerosis. Amyotroph Lateral Scler 2009;10:131-46.

3. Talman P, Forbes A, Mathers S. Clinical phenotypes and natural progression for motor neuron disease: analysis from an Australian database. Amyotroph Lateral Scler 2009;10:79-84.

4. Hardiman O, van den Berg LH, Kiernan MC. Clinical diagnosis and management of amyotrophic lateral sclerosis. Nat Rev Neurol 2011;7:639-49.

5. Neudert $\mathrm{C}$, Oliver D, Wasner $\mathrm{M}$, et al. The course of the terminal phase in patients with amyotrophic lateral sclerosis. J Neurol 2001;248:612-16.

6. Mioshi E, Lillo P, Yew B, et al. Cortical atrophy in ALS is critically associated with neuropsychiatric and cognitive changes. Neurology 2013;80:1117-23.

7. Lillo $\mathrm{P}$, Mioshi E, Zoing MC, et al. How common are behavioural changes in amyotrophic lateral sclerosis? Amyotroph Lateral Scler 2010;12:45-51.

8. Lillo P, Savage S, Mioshi E, et al. Amyotrophic lateral sclerosis and frontotemporal dementia: a behavioural and cognitive continuum. Amyotroph Lateral Scler 2012;13:102-9.

9. Rooney J, Byrne S, Heverin M, et al. A multidisciplinary clinic approach improves survival in ALS: a comparative study of ALS in Ireland and Northern Ireland. J Neurol Neurosurg Psychiatr 2015;86:496-501.

10. Hardiman O. Multidisciplinary care in motor neurone disease. In: Kiernan MC, ed. The motor neurone disease handbook. Sydney: MJA Books, 2007:164-74.

11. Hogden A, Greenfield D, Nugus P, et al. What are the roles of carers in decision-making for amyotrophic lateral sclerosis multidisciplinary care? Patient Prefer Adherence 2013;7:171-81.

12. Mitsumoto $\mathrm{H}$. Patient choices in ALS: life-sustaining treatment versus palliative care? Neurology 1999;53:248-9.

13. Hogden A, Greenfield D, Nugus $P$, et al. Development of a model to guide decision making in amyotrophic lateral sclerosis multidisciplinary care. Health Expect 2015;18:1769-82.

14. Hogden A, Greenfield D, Nugus $P$, et al. Engaging in patient decision-making in multidisciplinary care for amyotrophic lateral sclerosis: the views of health professionals. Patient Prefer Adherence 2012;6:691-701.

15. Hogden A, Greenfield D, Nugus $P$, et al. What influences patient decision-making in amyotrophic lateral sclerosis multidisciplinary care? A study of patient perspectives. Patient Prefer Adherence 2012;6:829-38.

16. Walsh T, Barr PJ, Thompson R, et al. Undetermined impact of patient decision support interventions on healthcare costs and savings: systematic review. BMJ 2014;348:g188.

17. Lang A, Toon L, Cohen SR, et al. Client, caregiver, and provider perspectives of safety in palliative home care: a mixed method design. Saf Health 2015;1:3.

18. Stacey D, Bennett CL, Barry MJ, et al. Decision aids for people facing health treatment or screening decisions. Cochrane Database Syst Rev 2011;(10):CD001431.

19. Elwyn G, Frosch D, Thomson R, et al. Shared decision making: a model for clinical practice. J Gen Intern Med 2012;27:1361-7.

20. Sivell S, Edwards A, Manstead AS, et al. Increasing readiness to decide and strengthening behavioral intentions: evaluating the impact of a web-based patient decision aid for breast cancer treatment options (BresDex: http://www.bresdex.com). Patient Educ Couns 2012;88:209-17.

21. Alden DL, Friend J, Schapira M, et al. Cultural targeting and tailoring of shared decision making technology: a theoretical framework for improving the effectiveness of patient decision aids in culturally diverse groups. Soc Sci Med 2014;105:1-8.

22. Katz SJ, Belkora J, Elwyn G. Shared decision making for treatment of cancer: challenges and opportunities. J Oncol Pract 2014:10:206-8.

23. Bhavnani V, Fisher B. Patient factors in the implementation of decision aids in general practice: a qualitative study. Health Expect 2010;13:45-54

24. Witteman HO, Dansokho SC, Colquhoun $\mathrm{H}$, et al. User-centered design and the development of patient decision aids: protocol for a systematic review. Syst Rev 2015;4:11. 
25. Volk RJ, Llewellyn-Thomas H, Stacey D, et al. Ten years of the International Patient Decision Aid Standards Collaboration: evolution of the core dimensions for assessing the quality of patient decision aids. BMC Med Inform Decis Mak 2013;13 (Suppl 2):S1.

26. Miller RG, Jackson CE, Kasarskis EJ, et al. Practice parameter update: the care of the patient with amyotrophic lateral sclerosis: drug, nutritional, and respiratory therapies (an evidence-based review): report of the Quality Standards Subcommittee of the American Academy of Neurology. Neurology 2009;73:1218-26.

27. Miller RG, Jackson CE, Kasarskis EJ, et al. Practice parameter update: the care of the patient with amyotrophic lateral sclerosis: multidisciplinary care, symptom management, and cognitive/ behavioral impairment (an evidence-based review): report of the Quality Standards Subcommittee of the American Academy of Neurology. Neurology 2009;73:1227-33.

28. Oliver D, Campbell C, Sykes N, et al. Decision-making for gastrostomy and ventilatory support for people with motor neurone disease: variations across UK hospices. $J$ Palliat Care 2011;27:198-201.

29. Mitsumoto $\mathrm{H}$, Bromberg $\mathrm{M}$, Johnston $\mathrm{W}$, et al. Promoting excellence in end-of-life care in ALS. Amyotroph Lateral Scler 2005;6:145-54.
30. Fletcher AJ, Marchildon GP. Using the Delphi method for qualitative, participatory action research in health leadership. Int $J$ Qual Methods 2014;13:1-18.

31. Elwyn G, Kreuwel I, Durand MA, et al. How to develop web-based decision support interventions for patients: a process map. Patient Educ Couns 2011;82:260-5.

32. Coulter A, Stilwell D, Kryworuchko J, et al. A systematic development process for patient decision aids. BMC Med Inform Decis Mak 2013;13(Suppl 2):S2.

33. Elwyn G, O'Connor A, Stacey D, et al. Developing a quality criteria framework for patient decision aids: online international Delphi consensus process. BMJ 2006;333:417.

34. O'Connor AM. Using decision aids to help patients navigate the "grey zone" of medical decision-making. CMAJ 2007;176:1597-8.

35. Flynn D, Nesbitt DJ, Ford GA, et al. Development of a computerised decision aid for thrombolysis in acute stroke care. BMC Med Inform Decis Mak 2015;15:6.

36. O'Connor AM. Ottowa Decision Support Framework to address decisional conflict. Secondary Ottowa Decision Support Framework to address decisional conflict 2006. http://www.ohri.ca/decisionaid (accessed Oct 2015).

37. Albrecht UV. Transparency of health-apps for trust and decision making. J Med Internet Res 2013;15:e277. 